
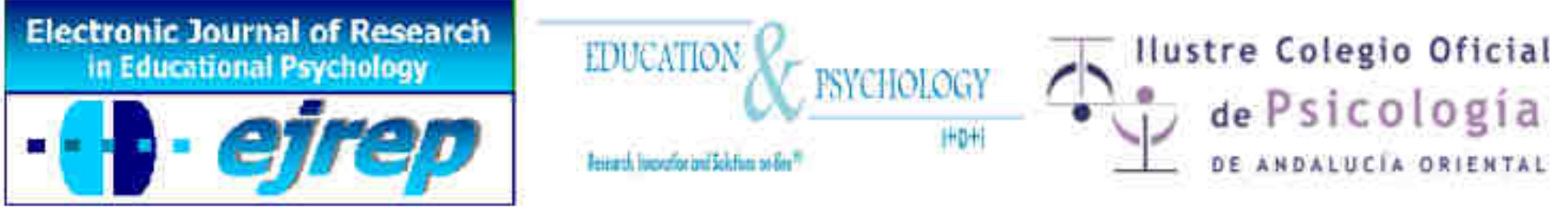

\title{
Revisores 2016
}


Adrián, Leonardo. Universidad de Córdoba (Argentina)

Ahmed, Wondimu. University of Groningen (The Nederlnads)

Al-Qaryouti, Ibrahim. Sultan Qabos Univerity (Sultanate of Oman)

Andres, Soledad Universidad de Angers (France)

Awoffala, Adeneye O.A. University of Lagos (Nigeria)

Ester Ato. Universidad de Murcia (Spain)

Amate, Jorge. Profesor de Educación Infantil. University of Almería (Spain)

Boatto, Yanina. Universidad Nacional de Río Cuato (Argentina)

Bred, Betty. Nord West University (South Africa)

Calero, Maria Dolores. Universidad de Granada (Spain)

Carnero Pinto, Joanna. University of Minho (Portugal)

Castejón, Juan Luis- Uuniversidad de Alicante (Spain)

Cedeño, Luis, Universidad Complutense de Madrid (Spain)

Dilmaç, Bullent. Necmettin Erbakan University Selçuklu (Turkey)

Fabber, Günter. University Hannover (Germany)

Faviery, Gladis. Universidad Tecnológica Nacional (Argentina)

Félix Mateo, Vicente. Hospital Clínico de Valencia (Spain)

Fernández, Javier. Universidad de Málaga (Spain)

Ferrando, María. University of Murcia (Spsin)

García Nicasio, Jesús. Universidad de León (Spain)

García Ross, Rafael. University of Valencia (Spain)

Guerreo, Eloisa. Universidad de Extremadura (Spain)

González-Torres, Mari Carmen. Universidad de Navarra (Spain)

Henao, Gloria Cecilia. Universidad de Buenaventura, Medellín (Colombia)

Hinz, Arnold. University of Education, Ludwigsburg (Germany)

Jiménez, Virginia. Universidad Complutense de Madrid (Spain)

Jones, Brett D. Virginia Tech (USA)

León, Benito. Universidad de Extremadura (Spain)

López, Magdalena. CONICET (Argentina)

Martín Lobo, Pilar. Universidad Internacional de la Rioja (Spain)

McLauhglin, T. F. Gonzaga University (USA)

Méndez, Marta. Universidad de Oviedo (España)

Michaelides, Michalis. European University Cyprus (Grecia)

Muñiz, José. Universidad de Oviedo (Spain) 
Oliveira, Helia. Institute of Education (Portugal)

Ordaz, Gabriela. Universidad Nacional Autónoma de México (México)

Pasmore, Grahamm. Lakeheat University (Canada)

Peralta Sánchez, F.J. University of Almería (Spain)

Ponce, Hector. Universidad Santigo de Chile (Chile)

Pepper, Debla. York University. Toronto (Canada)

Pichardo, María del Carmen. Universidad de Granada (Spain)

Prieto, María Dolores. Universidad de Murcia (Spain)

Roderer, Thomas. University of Bern (Switzerland)

Rosser, Ana María. Univesidad de Alicante (Spain)

Rouis, Sana. Lulea University Techology (Sweeden)

Rucillo, Maria Teresa. Universidad de Jaén (Spain)

Phan, Huy. University of South Pacific (Australia)

Shapiro, Amy. University of Massachuseth (USA)

Saéz Rodríguez, Alberto. Dyslexia Institute Genova (Switzerland)

Sáinz, Consuelo. Universidad de Burgos (Spain)

Sánchez, Oscar. Universidad de Murcia (Spain)

Schoor, Cornelia. University of Bamberg (Germany)

Segovia, Isidoro. Universidad de Granada (Spain)

Spinoza, Evelyn. University of Guatemala Valey (Guatemala)

Stephanou, Georgia. University of Wester Macedonia (Greece)

Trianes, María Victoria. Universidad de Málaga (Spain)

Lourdes Villardon, University of Deusto (Spain)

Vera, Manuel Mariano. Universidad de Granada (España)

Wass, Cristopher. Kent State University (USA) 
META-DATOS 2016

Recibidos: 210 articles

Rechazo inicial: 99 articles

Rechazo Final: 81 articles

Aceptados: 30 articles

Tasa de aceptación: $14.28 \%$

Tasa de Rechazo: $85.72 \%$ 